Int. J. Morphol.,

35(1):310-318, 2017.

\title{
Proteína Morfogenética Ósea y su Opción como Tratamiento de la Fisura Alveolar
}

\author{
Bone Morphogenetic Protein and its Option as an Alveolar Cleft Treatment
}

Francisca Uribe ${ }^{1,2,3}$; Mario Cantín ${ }^{4} ;$ Juan Pablo Alister ${ }^{1,2,5}$; Cristián Vilos ${ }^{6,7}$; Rodrigo Fariña ${ }^{8,9}$ \& Sergio Olate S1, $^{10}$

URIBE, F.; CANTÍN, M.; ALISTER, J.P.; VILOS, C.; FARIÑA, R. \& OLATE, S. Proteína morfogenética ósea y su opción como tratamiento de la fisura alveolar. Int. J. Morphol., 35(1):310-318, 2017.

RESUMEN:La proteína morfogenética ósea (BMP), es una proteína endógena que ha mostrado efectos significativos en la promoción de la formación ósea. El uso de BMP ha sido descrito en la reconstrucción de defectos óseos de origen traumáticos y patológicos, incluyendo la fisura alveolar, el aumento de reborde alveolar, la elevación de seno maxilar, el injerto de alveolo post-extracción, y la cirugía perimplantaria entre otros. A pesar de las ventajas asociadas al uso de BMP y que en la actualidad se aplica en combinación con matrices de colágeno, ciertas propiedades tales como su baja resistencia mecánica y su elevada tasa de liberación inicial disminuyen su eficacia en la formación ósea. En este contexto, el desarrollo de nuevos sistemas de liberación prolongada de BMP que permitan la quimiotaxis de células mesenquimáticas y su posterior diferenciación a osteoblastos representa un desafío con alto potencial clínico para la estimulación de la formación ósea. En este trabajo, se describe el uso de BMP en la reconstrucción de fisuras alveolares y en particular se discuten las ventajas de su administración en micropartículas poliméricas como sistemas de liberación de BMP (rhBMP-2) con promisorias aplicaciones en la estimulación de la formación ósea.

PALABRAS CLAVE: Proteína morfogenética ósea; rhBMP-2; Fisura alveolar; Micropartículas.

\section{INTRODUCCIÓN}

Las proteínas morfogenéticas óseas (BMPs) son un conjunto de proteínas endógenas que pertenecen a la familia de factor de crecimiento transformante beta (TGF- $\beta$ ) y que tienen la capacidad de inducir formación del hueso, cartílago y el tejido conectivo (Carreira et al., 2014a).

Las BMPs fueron descritas por el Dr. Marshal Urist en 1965 (Urist, 1965), al implantar matriz ósea desmineralizada extraída de bovino a nivel intramuscular en ratas y conejos y observar la producción ósea ectópica en el sitio de implantación; él lo denominó formación ósea por autoinducción. Urist atribuyó el proceso a la presencia de una proteína que atrae células mesenquimáticas pluripotenciales e induce formación ósea local, por lo que la denominó proteína morfogenética ósea (BMP).
Actualmente, se han identificado 20 tipos distintos de BMP, sin embargo sólo la BMP-2, BMP-7 y BMP-9 son capaces de promover la osteoinducción. Pese a que BMP es una proteína endógena que puede ser obtenida de tejido óseo desmineralizado, su aislamiento es inviable debido a que se encuentra en mínimas cantidades (Wozney et al., 1988). Estudios descritos en bovinos mostraron que es posible obtener $57 \mathrm{mg}$ de un extracto rico en BMPs por kilo de hueso fresco. Sin embargo, luego de realizar los procesos de purificación y aislamiento de una BMP en específico, el rendimiento se redujo a unos cuantos ng de BMP por kg de hueso (Carreira et al., 2014a).

Por otra parte, basados en las posibles reacciones inmunológicas que pueden ocasionar la utilización de teji-

\footnotetext{
${ }^{1}$ División de Cirugía Oral y Maxilofacial, Universidad de La Frontera, Temuco, Chile.

${ }^{2}$ Programa Doctorado en Ciencias Médicas, Facultad de Medicina, Universidad de La Frontera, Temuco, Chile.

${ }^{3}$ Becario CONICYT-PCHA/Doctorado Nacional/ 2015-21150752.

${ }^{4}$ Programa de Doctorado en Ciencias Morfológicas, Facultad de Medicina, Universidad de La Frontera, Temuco, Chile.

${ }^{5}$ Becario CONICYT-PCHA/Doctorado Nacional/2015-21150598.

${ }^{6}$ Universidad Andres Bello, Laboratory of Nanomedicine and Targeted Delivery, Center for Integrative Medicine and Innovative Science (CIMIS), Faculty of

Medicine; and Center for Bioinformatics and Integrative Biology (CBIB), Faculty of Biological Sciences, Santiago, Chile

${ }^{7}$ Center for the Development of Nanoscience and Nanotechnology, CEDENNA, 9170124, Santiago, Chile.

${ }^{8}$ Departamento de Cirugía y Traumatología Maxilofacial, Facultad de Odontología, Universidad de Chile, Santiago, Chile.

${ }^{9}$ Unidad de Cirugía Maxilofacial, Hospital del Salvador, Santiago, Chile.

${ }^{10}$ Centro de Investigación en Ciencias Biomédicas, Universidad Autónoma de Chile, Chile.
} 
do óseo bovino (Carreira et al., 2014a), en la década de los 80 , se logró adquirir la secuencia de homólogos humanos de BMP bovina, las cuales se introdujeron en células mamíferas para inducir la secreción de BMP. Esta herramienta de recombinación genética permitió generar una mayor concentración de BMP y de mejor calidad en términos de su homogeneidad (Wozney et al.). Actualmente, las proteínas morforgenéticas recombinantes humanas (rhBMP) más estudiadas y utilizadas son los tipos rhBMP-2 y rhBMP-7 (Davies et al., 2010).

En este contexto, el objetivo de este trabajo de revisión consiste en discutir uso de BMP en reconstrucción ósea, principalmente orientada a pacientes con fisura labio palatina y establecer opciones de innovadoras estrategias de liberación de fármacos basadas en partículas formuladas de polímeros biodegradables y biocompatibles.

\section{Estructura molecular y actividad biológica}

Las proteínas morfogenéticas óseas en términos generales son moléculas diméricas constituidas por aproximadamente 120 aminoácidos, siendo una secuencia de aminoácidos muy estable; se calcula que existe hace 600 millones de años (Rengachary, 2002). Todos los tipos de BMP presentan siete residuos de cisteína y la ubicación de éstos residuos son idénticos en todos los miembros de la familia de TGF- $\beta$. El segmento maduro se ha preservado en todos los organismos, seis de los residuos de cisteína son estabilizados por tres enlaces disulfuro formando una estructura molecular como "nudo de cisteína" (Wozney et al.; Rengachary; Carreira et al., 2014 b).

BMP es producida en el tejido óseo por células osteoprogenitoras, osteoblastos, condrocitos, plaquetas y células endoteliales. Posterior a la formación de BMP, ésta se almacena temporalmente en la matriz extracelular durante periodos de reparación y remodelación ósea (Carreira et al. b). La actividad biológica de la BMP ocurre mediante la formación de estímulos que desencadenan una formación ósea endocondral. El mecanismo de acción de las BMP es mediado por la quimiotaxis de monocitos, los cuales producen TGF- $\beta$ y a su vez promueven la quimiotaxis y proliferación de células mesenquimáticas (Wozney et al.; Davies et al.). BMP induce la diferenciación de éstas células a condrocitos, dicho fenómeno es seguido por una hipertrofia de los condrocitos, calcificación de la matriz ósea, diferenciación de osteoblastos y finalmente la formación ósea. La calidad del tejido óseo formado con BMP es indistinguible del tejido óseo formado sin BMP; sin embargo, las diferencias radican en la velocidad y en la dirección de la formación del tejido óseo. El tejido óseo formado con BMP es capaz de cicatrizar en menor tiempo. El periodo normal de cicatrización de un individuo sano tarda entre 12 a 16 semanas lo cual es significativamente mayor que un tejido óseo expuesto a BMP que tarda en cicatrizar entre de 8 a 10 semanas.

La direccionalidad de la formación del tejido óseo en una brecha en ausencia de BMP se desarrolla desde los márgenes hacia el centro, mientras que al utilizar BMP la formación ósea se produce en diferentes zonas del defecto al mismo tiempo (Rengachary).

La función osteoinductiva de la BMP depende directamente de la dosis utilizada. Dosis bajas generan cartílago y poca cantidad de tejido óseo en cambio mayores concentraciones generan mayor cantidad de tejido óseo y puede resultar mediante osificación directa o membranosa (Urist; Wozney et al.; Davies et al.). Por ésta razón la concentración de BMP en el sitio del injerto es más importante que la dosis total administrada, por lo que la dosis terapéutica sugerida para humanos de rhBMP-2 es de $1,5 \mathrm{mg} / \mathrm{ml}$ de suero fisiológico (Davies et al.). Boyne et al., (2005) demostraron que la dosis de rhBMP-2 de $1,5 \mathrm{mg} / \mathrm{ml}$ es segura para la utilización en elevación de seno maxilar en humanos; en animales las dosis requeridas son menores, planteándose dosis de 0,01 a $0,1 \mathrm{mg} / \mathrm{ml}$ en ratas, $0.4 \mathrm{mg} / \mathrm{ml}$ en conejos y 1 a $1,5 \mathrm{mg} / \mathrm{ml}$ en monos (Li et al., 2001).

La concentración ideal para inducir el cierre de un defecto óseo depende de diferentes factores (Rengachary). En primer lugar la escala de evolución ya que una rata cicatriza más rápido que una forma más avanzada como los primates, por lo que mientras más avanzados en la escala de evolución, requieren más dosis de BMP. También se debe considerar el tamaño del defecto ya que si los bordes están en contacto no es necesario el uso de BMP exógena y la liberación de BMP endógena seria suficiente para la cicatrización ósea. Cuando existe un defecto óseo crítico es necesario el aporte de BMP, mientras mayor sea el defecto, se requiere mayor dosis de BMP (Rengachary). La dosis también puede variar por la vascularización de la zona y la cantidad de células mesenquimáticas cercanas al defecto ( $\mathrm{Li}$ et al.).

En marzo 2007 la FDA autorizó el uso de rhBMP-2 INFUSE (Medtronic Sofamor Danek, Minneapolis, MN) como alternativa a injertos autólogos en elevación de seno maxilar y para aumento de reborde alveolar asociado a alveolos post-extracción. En año 2012 se reportaron las complicaciones por el uso de INFUSE en la base de datos de la FDA (Woo, 2012); se presentaron 83 complicaciones en el territorio maxilofacial $(8,9 \%$ del total de las complicaciones). De éstas complicaciones sólo el 33,7 \% se utilizaron según las indicaciones aprobadas por la FDA. Las otras complicaciones se presentaron durante reconstrucción de man- 
díbula por fractura o cáncer $(30,1 \%$,$) , reconstrucción de$ fisura alveolar $(22,9 \%)$ y otras cirugías maxilofaciales no especificadas $(13,3 \%)$. Las complicaciones reportadas fueron reacciones locales como edema, eritema o dolor, infección del sitio quirúrgico o complicaciones de la herida, fracaso del injerto o pseudoartrosis. De todas las complicaciones el 30,1\% reportó que el paciente requirió de una cirugía adicional para solucionar el problema.

El uso de rhBMP-2 en la región cráneofacial es extenso; se han realizado estudios en animales y humanos con múltiples aplicaciones clínicas. Su aplicación se puede considerar en la reconstrucción ósea de defectos de origen traumático, patológico o congénito como por ejemplo en la reconstrucción de la fisura alveolar. Además se puede utilizar en cirugía dentoalveolar en aumento de reborde alveolar, elevación de seno maxilar, injerto de alveolo post-extracción, cirugía perimplantaria entre otros.

En relación a la fisura alveolar, existen numerosos estudios en animales donde se realiza una fisura alveolar quirúrgica en monos (Boyne et al., 1998), perros (Mayer et al., 1996; Kawamoto et al., 2002), conejos (el-Bokle et al., 1993) o ratas (Nguyen et al., 2009; Nampo et al., 2010; Mostafa et al., 2014; Xu et al., 2015), en donde se crea un defecto óseo crítico para el estudio de los injertos de la fisura alveolar en humanos. Se han comparado rhBMP-2 con distintos tipos de injertos o también se han estudiado distintas matrices para llevar la rhBMP-2 al defecto óseo (Tabla I).

Se reportó una serie de casos en donde utiliza solo rhBMP-2 en la reconstrucción de la fisura alveolar en humanos (Chin et al., 2005). Se obtuvo una reconstrucción ósea exitosa en 49/50 fisuras en pacientes de 6 a 14 años. En las fisuras severas se realizó una combinación de distracción osteogénica con rhBMP-2 pero no se utilizó injerto autólogo. El tejido óseo obtenido tuvo características clínicas normales en términos de erupción de piezas dentarias, en movimientos ortodóncicos y en las características histológicas.

En el año 2007 se realizó un estudio en donde utilizó rhBMP-2 en 10 fisuras alveolares y las comparó con injerto de cresta ilíaca de dos fisuras alveolares (Herford et al., 2007). Se midió el volumen óseo formado mediante tomografía computarizada. El volumen óseo de las fisuras reconstruidas mediante rhBMP-2 fue de $71,7 \%$, en cambio en el grupo de autoinjerto fue de 78,1\%. Los autores plantean que probablemente el grupo de rhBMP-2 fue inferior debido a que el seguimiento fue sólo de 4 meses o por una falla en la técnica quirúrgica en el cierre del plano nasal. Sin embargo concluyen que la rhBMP-2 es una alternativa efectiva al injerto autólogo de cresta iliaca.
Luego en el año 2008 se realizó un ensayo clínico en 21 pacientes esqueletalmente maduros (Dickinson et al., 2008). Los grupos fueron elegidos al azar entre el uso de rhBMP-2 e injerto autólogo de cresta ilíaca; el seguimiento fue por 1 año. El grupo de rhBMP-2 tuvo menos complicaciones $(11 \% \mathrm{v} / \mathrm{s} 50 \%)$, mayor mineralización del tejido óseo, mayor ocupación del defecto óseo (95 \% v/s $63 \%$ ). Por lo tanto los autores concluyen que el uso de rhBMP-2 mejora la formación ósea y disminuye la morbilidad del procedimiento en comparación con el autoinjerto de cresta ilíaca. En el año 2010, se realizó un estudio similar en 16 pacientes en crecimiento ( 8 a12 años) (Alonso et al., 2010). Las complicaciones por dolor fueron más frecuentes en el grupo de autoinjerto de cresta ilíaca $(87,5 \%$ de los pacientes presentaban dolor a los 14 días). La ocupación del defecto óseo a los 6 meses fue significativamente mayor en el grupo de autoinjerto ( $75,4 \% \mathrm{v} / \mathrm{s} 59,6 \%)$, pero a los $12 \mathrm{me}$ ses no había diferencia significativa. La altura ósea fue significativamente mayor en el grupo de autoinjerto de cresta ilíaca a los 12 meses $(86,8 \%$ v/s $65 \%)$ y la erupción dentaria se desarrollo con normalidad en ambos grupos. Los autores describen que la altura ósea disminuida en el grupo de rhBMP-2 se presentó en la zona cercana a la piriforme y se puede explicar por la falta de estabilidad de la matriz (esponja de colágeno tipo I) o la presencia de tejido blando y la mal posición del cornete inferior. Los autores concluyen que mediante el uso de rhBMP-2 en fisuras alveolares se obtiene un resultado satisfactorio con una adecuada erupción dentaria y menor morbilidad en comparación con autoinjerto de cresta ilíaca.

Posteriormente, en el año 2012 se realizó un ensayo clínico en 18 pacientes en crecimiento ( 8 a 15 años) divididos en tres grupos al azar; periostioplastía, autoinjerto de cresta iliaca y rhBMP-2 con seguimiento de 1 año (Canan et al., 2012). La formación de tejido óseo fue similar en el autoinjerto (78 \%) y en el grupo de rhBMP-2 $(75,1 \%)$ y significativamente mayor formación ósea que el grupo de periostioplastía.

Es posible que la matriz que se utiliza (esponja de colágeno) no sea la más adecuada ya que presenta una baja resistencia generando un colapso de la matriz a medida que se reabsorbe, además presenta una liberación de rhBMP-2 inicial aumentada promoviendo la inflamación local (Carreira et al. 2014b). Adicionalmente, al no tener una liberación controlada y prolongada obliga a utilizar mayores dosis para poder obtener una formación ósea adecuada (Carreira et al. 2014b). En el año 2013 se realizó un estudio retrospectivo utilizando fichas clínicas por 2 años donde se obtuvieron 55 pacientes con un promedio de edad de 11,8 años y 21 meses de seguimiento (Francis et al., 2013). Se compararon 36 pacientes $(65 \%)$ en los que se utilizó rh- 


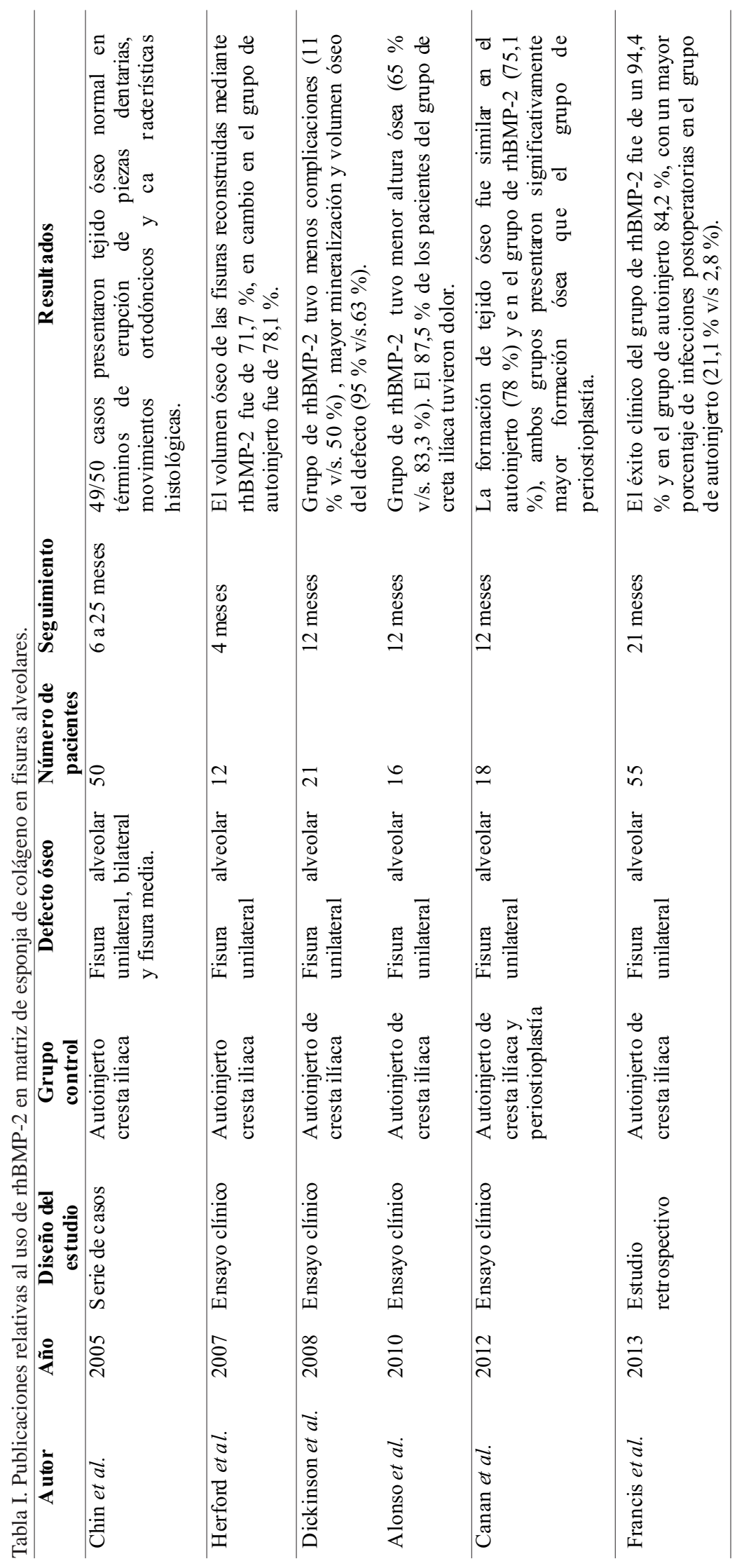

BMP-2 con matriz ósea desmineralizada y 19 pacientes $(35$ $\%)$ en los que se realizó injerto de cresta ilíaca. El éxito clínico del grupo de rhBMP-2 fue de un $94,4 \%$ y en el grupo de autoinjerto $84,2 \%$, con un mayor porcentaje de infecciones postoperatorias en el grupo de autoinjerto (21,1\% v/s 2,8\%); el grupo de rhBMP-2 fue superior en relación a la formación ósea. Los autores plantean que el uso de la esponja de colágeno tipo I es insuficiente y debería ser utilizada con matriz ósea desmineralizada ya que provee una matriz osteoconductiva para formación ósea y actúa como una resistencia que previene el colapso del tejido blando.

Se han estudiado diferentes alternativas de matrices para utilizar rhBMP-2. Las matrices inorgánicas como la HA o el $\beta$-TCP por ejemplo, presentan como principal ventaja una excelente resistencia mecánica pero no son biodegradables (Li et al.). Se han utilizado en conjunto con polímeros naturales o sintéticos mejorando la biocompatibilidad y resistencia de éstos (Li et al.). También existe la alternativa de utilizar polímeros sintéticos en donde el ácido poli-láctico-co-poli-glicólico (PLGA) es el más utilizado por ser biocompatible, tener una gran variedad de presentaciones y mayor resistencia que los polímeros naturales $(\mathrm{Li}$ et al.). Es necesario estudiar nuevas matrices para utilizar menores dosis pero con una liberación controlada y prolongada en el tiempo y así evitar estos efectos adversos.

Otro aspecto importante es el elevado costo de la rhBMP-2. En el año 2014, se realizó un estudio retrospectivo de 3478 pacientes a los que se les realizó injerto alveolar, de los cuales un 79,4\% de los casos se realizó con injerto de cresta ilíaca y un 1 $\%$ con rhBMP-2 (Allareddy, 2014). El uso de rhBMP-2 aumentó los costos 
significativamente, sin embargo otro estudio en el año 2008, también comparó los costos entre ambos procedimientos concluyendo que el grupo de rhBMP-2 presenta menor tiempo de hospitalización, menor morbilidad y menor costo final del procedimiento (USD $\$ 11.100 \mathrm{v} / \mathrm{s}$ USD $\$ 21.800$ ) (Dickinson et al.). Por otra parte, aunque los resultados clínicos presentados son positivos, los estudios pueden estar sesgados ya que el número de pacientes utilizado es bajo, el estudio que tenía el mayor tamaño de muestra fue 55 sujetos (Francis et al.).

\section{Micropartículas Poliméricas}

La administración clásica de fármacos que poseen una limitada solubilidad conlleva el uso o administración de elevadas dosis y/o dosis repetitivas para alcanzar una concentración adecuada y lograr el efecto terapéutico. Ésta modalidad puede generar fluctuaciones significativas en el torrente sanguíneo que provocan mayores reacciones adversas a medicamentos o incluso llegar a dosis tóxicas. Éstos inconvenientes han llevado al desarrollo de nuevas estrategias de tratamiento más efectivas y con menos efectos colaterales para los pacientes como la liberación prolongada o sostenida de fármacos (Vilos et al., 2012). En particular, en la regeneración ósea, las principales limitaciones asociadas a estrategias regenerativas incluyen la alteración de la proliferación y diferenciación celular, la solidez estructural insuficiente de las matrices y una baja dosis de factor de crecimiento necesaria para una osteogenesis eficiente (Walmsley et al., 2015).

Las micropartículas poliméricas (MP) son una alternativa que se ha utilizado en la liberación controlada de antibióticos, anti-inflamatorios y quimioterapéuticos, etc . MPs ofrecen la ventaja de alcanzar el efecto terapéutico deseado con un menor número de dosis y frecuencia de administración, por lo tanto, su aplicación reduce el número y frecuencia de reacciones adversas y promueve la liberación prolongada en el tiempo (Vilos et al., 2014) permitiendo a su vez la diferenciación de las células osteoprogenitoras a osteoblastos y por consecuencia la osteogenesis (Li et al.; Vo et al., 2012). Asimismo, el uso de MPs permite encapsular y liberar en forma sostenida fármacos lábiles, con baja solubilidad y altamente tóxicos (Vilos et al., 2015).

En el presente, es posible desarrollar transportadores de fármacos basados tanto micro como en nanopartículas (NP) poliméricas, donde su principal diferencia es el tamaño. MP tienen un tamaño de 0,1 a $100 \mu \mathrm{m}$ y las NP de 0,1 a $100 \mathrm{~nm}\left(1 \mathrm{~nm}=1 \times 10^{-6} \mathrm{~mm}\right)$ (Riehemann et al., 2009; Kamaly et al., 2016). Esta diferencia en su escala de tamaño se torna altamente significativa al comparar la superficie de contacto que representa cada sistema. Asimismo, esto gene- ra un gran impacto en el comportamiento de las partículas tanto en su entorno biológico como fisiológico. La relación entre el área y el volumen o densidad de superficie $\left(\mathrm{mm}^{2} /\right.$ $\mathrm{mm}^{3} \mathrm{o} \mathrm{nm} / \mathrm{nm}^{3}$ ) es inversamente proporcional al radio de la partícula, es decir, las NP que tienen un radio menor, poseen una mayor relación entre el área y el volumen, obteniendo mayor contacto con el medio a través de su superficie. Las MP en cambio, al tener un radio mayor, presentan una relación entre área y volumen menor y tienen menor contacto con el medio a través de su superficie (Kohane, 2007; Kumari et al., 2010).

La comparación entre ambos sistemas es equívoca al valorar uno más que otro, ya que su aplicación va a depender exclusivamente de la estrategia terapéutica. Por ejemplo, las MP se utilizan preferencialmente para la liberación prolongada y sostenida de fármacos a nivel local, y mantener el principio activo en el sitio de acción. Las NP por su parte, se utilizan como estrategia de liberación de fármacos a nivel intracelular, donde el objetivo fundamental es lograr la liberación en organelos o en sitios específicos de la célula. En el contexto de la reconstrucción ósea, el uso de MP presenta múltiples ventajas, considerando la necesidad de contar con un estímulo permanente en el tejido óseo. Los polímeros se clasifican y describen en diferentes familias (Pillai et al., 2001); polímeros naturales y polímeros sintéticos, los cuales pueden subdividirse en biodegradables y no biodegradables (Tabla II).

Los polímeros naturales presentan ventajas como baja toxicidad, bajos costos de manufactura, ser biodegradables y renovables. Al aplicarlos en la ingeniería de tejidos presentan señalización biológica, adhesión celular, respuesta celular y remodelación (Pillai et al.; Puppi et al., 2010). En términos globales, los polímeros naturales son hidrosolubles y se degradan rápidamente. Además, su uso es limitado debido a que, en los procedimientos de síntesis de partículas, pueden perder propiedades e incluso transformarse en un factor de riesgo capaz de activar la respuesta inmune. Estudios han mostrado el beneficioso efecto de la incorporación de modificaciones químicas en las cadenas poliméricas que permiten mejorar sus propiedades fisicoquímicas y estabilidad (Puppi et al.) por lo que estas desventajas hacen que los polímeros naturales sean menos atractivos que los polímeros sintéticos (Kamaly et al.).

Por otra parte, los polímeros sintéticos pueden ser producidos a gran escala con técnicas de síntesis química de alta precisión, permitiendo la conformación de co-polímeros y biomateriales altamente compatibles con respuesta inteligente a cambios de $\mathrm{pH}$, temperatura, potencial redox, etc. tienen como ventaja que disminuyen las posibilidades de reacciones de inmunogenicidad, toxicidad y transmisión de 
URIBE, F.; CANTÍN, M.; ALISTER, J.P.; VILOS, C.; FARIÑA, R. \& OLATE, S. Proteína morfogenética ósea y su opción como tratamiento de la fisura alveolar. Int. J. Morphol., 35(1):310-318, 2017.

Tabla II. Polímeros naturales y sintéticos utilizados en la liberación controlada de drogas empleadas actualmente.

\begin{tabular}{|c|c|c|}
\hline Polímero & Ventajas & Desventajas \\
\hline 1. Natural & $\begin{array}{l}\text { Baja toxicidad, bajo costos manufactura, biodegradables y } \\
\text { renovables. }\end{array}$ & $\begin{array}{l}\text { Biodegradación rápida, reacciones de } \\
\text { inmu nogenicidad, baja resistencia mecánica. }\end{array}$ \\
\hline - Colágeno & Baja antigenicidad, buena adhesión celular, porosidad. & $\begin{array}{l}\text { Rápida biodegradación y baja resistencia } \\
\text { mecánica. }\end{array}$ \\
\hline - Alginato & Biocompatible, biodegradable. & $\begin{array}{l}\text { Baja resi stencia mecánica, difícil manipulación } \\
\text { y esterilización. }\end{array}$ \\
\hline - Ácido Hialurónico & Biocompatible, buenas propiedades visco-elásticas. & Alta solubilidad, rápida biodegradación. \\
\hline - Dextrán & Buenas propiedades mecánicas & Difícil manipulación y esterilización. \\
\hline - Chitosán & $\begin{array}{l}\text { Promueve adhesión, proliferación y diferenciación celular. } \\
\text { Porosidad. }\end{array}$ & $\begin{array}{l}\text { Bajas propiedades mecánicas. Puede provocar } \\
\text { reacción a cuerpo extraño. }\end{array}$ \\
\hline 2. Sintéticos & $\begin{array}{l}\text { Sin reacciones de inmunogenicidad. Propiedades fís icas y } \\
\text { velocidad de biodegradación predecible y reproducible. }\end{array}$ & $\begin{array}{l}\text { Se pueden promover respuestas inflamatorias } \\
\text { no deseadas en la biodegradación. }\end{array}$ \\
\hline - PLGA & $\begin{array}{l}\text { Buenas propiedades mecánicas, tiempo de biodegradación } \\
\text { variable según la relación de PLA:PLG. }\end{array}$ & $\begin{array}{l}\text { Respuesta inflamatoria local por productos de } \\
\text { degradación. }\end{array}$ \\
\hline - Poli-_-capronolactona [PCL] & Bajas propiedades mecánicas. & Biodegradación muy lenta (años). \\
\hline - Poli-Uretanos [PU] & Buenas propiedades elásticas y mecánicas. & $\begin{array}{l}\text { Productos de degradación pueden provocar } \\
\text { reacción inflamatoria local. }\end{array}$ \\
\hline - Pluronic & $\begin{array}{l}\text { Propiedad termo-gelificante, fácil manipulación, baja } \\
\text { toxicidad. }\end{array}$ & Propi edades mecánicas moderadas. \\
\hline
\end{tabular}

enfermedades (Puppi et al.; Vo et al.). Sin embargo la falta de biocompatibilidad puede promover respuestas celulares no deseadas; aun así, son predecibles y reproducibles en sus resultados. Dicha respuesta "smart" permite determinar la velocidad de degradación y consecuentemente ajustar la tasa de liberación de los fármacos, propiedades físicas, químicas, velocidad de degradación, se pueden fabricar en la forma y tamaño deseado siendo fáciles de esterilizar (Puppi et al.; Yameen et al., 2015).

Uno de los polímeros sintéticos biocompatibles más utilizados es el ácido poli-láctico-co-poli-glicólico (PLGA). El PLGA es un polímero sintético biodegradable comúnmente utilizado par fabricar materiales biomédicos como suturas, mallas, membranas, material de osteosíntesis, matrices tridimensionales entre otros, y se encuentra autorizado por la FDA. PLGA ofrece una amplia variedad de propiedades físicoquímicas dependiendo en sus características estructurales tales como peso molecular, razón ácido láctico - ácido glicólico, etc. El PLGA de alto peso molecular se puede utilizar para placas y tornillos de osteosíntesis debido a su solidez estructural, el PLGA de bajo peso molecular, en cambio, se utiliza principalmente en el desarrollo de sistemas de liberación controlada de fármacos (Pulapura et al., 1992).

La estructura química de PLGA conformada por enlaces de tipo éster permite su degradación mediante hidrólisis en ácido poli-glicólico (PGA) que es convertido en metabolitos y el ácido poli-láctico (PLA) que mediante el ciclo de Krebs se transforma en $\mathrm{CO}_{2}$ y $\mathrm{H}_{2} \mathrm{O}$. Asimismo, PLGA puede ser metabolizado por enzimas tales como hidrolasas, proteasas, glicosidadas y fosfatasas (Virlan et al., 2015). El PGA es un polímero hidrofílico con una tasa de degradación rápida, en cambio PLA tiene un grupo metilo que le entrega propiedades químicas, físicas y mecánicas más estables. El PLGA ofrece mejor control en la degradación que los polímeros de PLA y PGA en forma individual y se puede variar la proporción entre PLA y PGA para aumentar o reducir el tiempo de reabsorción. Sin embargo se ha descrito que la aplicación de PLGA puro como matriz para la regeneración ósea posee propiedades mecánicas sub-óptimas para poder soportar funciones de carga. Por ésta razón se ha utilizado en combinación con otros materiales como cerámicos o vidrio (Gentile et al., 2014). 


\section{Uso de rhBMP-2 encapsulado en MP de PLGA}

Se han descrito diferentes métodos para la síntesis de MP con fármacos hidrófilos e hidrofóbicos (Vilos et al. 2015). Estudios han demostrado que la liberación de rhBMP2 desde una MP de PLGA es afectada directamente por el peso molecular, la relación entre PLA y PGA, la naturaleza del grupo terminal y la cantidad de rhBMP-2 incorporada en la MP (Anderson et al.,2012). En el año 2001 se comparó el uso de MP de PLGA con rhBMP-2 en una matriz de carbometil-celulosa y la misma matriz con MP sin rhBMP-2 (Schrier et al., 2001). En este trabajo, se estudió el efecto de ambos tipos de partículas en un modelo de defecto óseo en calota de conejo. Luego de 6 semanas se observó que las MP de PGLA con rhBMP-2 tuvieron una formación ósea significativamente mayor que el grupo sin rhBMP-2. En el año 2005 se demostró que las MP de PLGA proveen de una liberación prolongada y adecuada de rhBMP-2 para la formación de un puente óseo en el cráneo de una rata (Ruhe et al., 2005).

En el año 2009, se utilizaron MP de PLGA de rhBMP2 en una matriz compuesta de chitosan/colágeno para evaluar su uso en la oseointegración de implantes dentarios en mandíbulas de perros (Shi et al., 2009). Se evaluaron dos grupos de matrices compuestas de chitosan/colágeno un grupo con MP de rhBMP-2 y el otro con rhBMP-2 sin MP. La liberación de rhBMP-2 fue evaluada in vitro, el grupo con MP tuvo una alta liberación de rhBMP-2 al inicio, se mantuvo en el tiempo y a los 21 días presentó una liberación menor. A las 6 horas el grupo con MP tuvo una liberación del $30 \%$ y el grupo sin MP del $50 \%$. A las 24 horas, el grupo con MP $<50 \%$ y el grupo sin MP $>60 \%$. A los 7 días el $80 \%$ de la rhBMP-2 se había liberado del grupo sin MP, en cambio en el grupo con MP el $80 \%$ de la liberación se alcanzó el día 21. El grupo con MP tuvo significativamente mayor tejido óseo en contacto con el implante, mayor densidad ósea y formación ósea dentro del implante que el grupo sin MP concluyendo que la presencia de MP prolonga la liberación de la rhBMP-2 en el tiempo permitiendo una formación ósea adecuada.

Posteriormente en el año 20015, se realizó una comparación entre MP de alginato y MP de PLGA con rhBMP-2 en matrices de colágeno/HA (Quinlan et al., 2015). Para ello, se realizaron estudios in vitro y in vivo en defectos óseos de 7 mm en cráneos de ratas. Los resultados obtenidos, mostraron que las MP de PLGA eran esféricas y porosas en comparación con las MP de alginato que mostraron una forma irregular con una superficie lisa. La liberación de la rhBMP-2 desde la MP de PLGA presentó una liberación acelerada inicial en el primer día, luego se mantuvo una liberación sostenida por los siguientes 14 días. Las MP de alginato, en cambio, presentaron una liberación acelerada durante los primeros 7 días seguido de una liberación más lenta y prolongada durante los siguientes 7 días; al finalizar, las MP de alginato y PLGA liberaron un $46 \%$ y $12 \%$ de rhBMP- 2 respectivamente obteniendo liberación de rhBMP-2 de ambos grupos, donde las MP de PLGA en matrices de colágeno/HA demostraron mayor capacidad osteogénica que las MP de alginato.

También se ha estudiado la liberación controlada de diferentes factores de crecimiento en una matriz reabsorbible de policaprolactona en defectos óseo de $3 \mathrm{~cm}$ en tibia de ovino (Kirby et al., 2016). En su diseño experimental, comparó un grupo de MP de rhBMP-2 con MP de un conjunto de factores de crecimiento; factor de crecimiento vascular endotelial (VRGF), factor de crecimiento derivado de plaquetas (PFGF) y rhBMP-2. Luego de 6 meses se evaluaron las propiedades mecánicas y la formación ósea sin encontrar diferencias significativas entre ambos grupos, concluyendo que el uso de rhBMP-2 como factor de crecimiento único es suficiente para asegurar una formación ósea adecuada.

En el año 2007, se comparó el uso de una matriz de HA y una matriz de $\beta$-TCP con y sin Pluronic, cargadas (rhBMP2) y vacías (rhBMP-2 libre), en defectos óseos críticos de calota de conejo (Zhou et al., 2007). A las 6 semanas se observó formación ósea en todos los grupos donde el Pluronic mejoró la manipulación de la matriz sin alterar su capacidad de promover la formación ósea. Al agregar rhBMP-2 a éstos grupos aumentó significativamente su capacidad osteogénica. Posteriormente, en el año 2008, se comparó el uso de rhBMP-2 en solución acuosa, rhBMP- 2 con esponja de colágeno, rhBMP-2 con Pluronic y rhBMP-2 con Pluronic y esponja de colágeno en un defecto óseo en el cuerpo mandibular de ratas Wistar (Issa $e t$ al., 2008). Los animales fueron sacrificados a las 2 y 4 semanas donde se observó que la rhBMP-2 tuvo diferencias significativas en la formación ósea al ser asociado con Pluronic, esponja de gelita y especialmente con la combinación de ambos.

Otros estudios, evaluaron la regeneración ósea en defecto óseo crítico de calota de rata a las 4 semanas utilizando rhBMP-2 en NP de PLGA en un gel de fibrina y Poloxamer (Chung et al., 2007). La regeneración ósea fue significativamente mayor que al utilizar el gel de fibrina, las NP de PGLA en un de gel de fibrina y Poloxamer sin rhBMP-2 o gel de fibrina y Poloxamer con rhBMP-2 no encapsulado. En el año 2012, se realizó un estudio para evaluar la regeneración ósea alveolar y periodontal en defectos óseos maxilares de cerdos (Chung et al., 2012). Se comparó un grupo de Pluronic con rhBMP-2 y células mesenquimáticas tratadas mediante terapia génica con un adenovirus que induce la formación endógena de rhBMP-2 con un grupo de Pluronic con células mesenquimáticas solas; a los 3 meses se observó un volumen óseo y regeneración periodontal significativamente mayor en las células tratadas genéticamente para producir rhBMP-2. 
Recientemente, se desarrolló una matriz de Pluronic con Poli-e-capronolactona (PCL) a la cual se le incorporó osteoblastos derivado del periostio, rhBMP-2 y/o factor de crecimiento vascular endotelial (VEGF) mediante heparinización (Lee et al., 2017). En este trabajo se compararon los grupos de rhBMP-2, VEGF y rhBMP-2 + VEGF en defectos óseos mandibulares en cerdos miniatura con un seguimiento de 2 años. El grupo de VEGF fue el que tuvo menor formación ósea, seguido por el grupo de rhBMP-2 + VEGF, el grupo rhBMP-2 fue el grupo que tuvo significativamente mayor formación ósea del defecto.

\section{CONCLUSIÓN}

La proteína rhBMP-2 presenta propiedades osteoinductivas, osteoconductivas y osteogénicas siendo una buena alternativa en reconstrucción ósea. El uso de rhBMP2 en la reconstrucción de defectos complejos como fisuras alveolares es promisorio, considerando el empleo de MP con rhBMP-2 una opción rentable tanto en aspectos biológicos como económicos.

URIBE, F.; CANTÍN, M.; ALISTER, J.P.; VILOS, C.; FARIÑA, R \& OLATE, S. Bone morphogenetic protein and its option as an alveolar cleft treatment. Int. J. Morphol., 35(1):310-318, 2017.

SUMMARY: Bone morphogenetic protein (BMP) is an endogenous protein that has shown significant effects in the promotion of bone formation. BMP also has been described in the reconstruction of traumatic and pathological bone defects, including alveolar cleft, alveolar ridge augmentation, maxillary sinus elevation, and applications in post-extraction alveolus graft, and peri-implant surgery among others. Despite the advantages associated with the use of BMP, currently is applied in combination with collagen matrices, which has certain properties such as low mechanical resistance and a high burst initial release that diminish its effectiveness in bone formation. In this context, the development of novel systems with greater mechanical resistance and prolonged release of BMP, that lead to chemotaxis of mesenchymal cells, following by its differentiation to osteoblasts represents a major challenge that holds outstanding clinical potential for the stimulation of bone formation. In this paper, we describe the use of BMP for the reconstruction of alveolar clefts, and its advantages being administrated in polymeric microparticles as sustain release system with promising applications in the stimulation of bone formation.

KEY WORDS: Bone morphogenetic protein; rhBMP-2; Alveolar cleft; Micropaticles.

\section{REFERENCIAS BIBLIOGRÁFICAS}

Allareddy, V. Use of human recombinant bone morphogenetic protein is associated with increased hospital charges in children with cleft lip and palate having bone graft procedures. J. Oral Maxillofac. Surg, 72(12): 2531-8, 2014

Alonso, N.; Tanikawa, D. Y.; Freitas Rda, S.; Canan, L., Jr.; Ozawa, T. O. \& Rocha, D. L. Evaluation of maxillary alveolar reconstruction using a resorbable collagen sponge with recombinant human bone morphogenetic protein-2 in cleft lip and palate patients. Tissue Eng. Part C Methods, 16(5): 1183-9, 2010.

Anderson, J. M. \& Shive, M. S. Biodegradation and biocompatibility of pla and plga microspheres. Adv. Drug Deliv. Rev., 64:72-82, 2012.

Boyne, P. J.; Nath, R., \& Nakamura, A. Human recombinant bmp-2 in osseous reconstruction of simulated cleft palate defects. Br. J. Oral Maxillofac. Surg., 36(2): 84-90, 1998.

Boyne, P. J.; Lilly, L. C.; Marx, R. E.; Moy, P. K.; Nevins, M.; Spagnoli, D. B., \& Triplett, R. G. De novo bone induction by recombinant human bone morphogenetic protein-2 (rhbmp-2) in maxillary sinus floor augmentation. J. Oral Maxillofac. Surg., 63(12):1693-707, 2005.

Canan, L. W., Jr.; da Silva Freitas, R.; Alonso, N.; Tanikawa, D. Y.; Rocha, D. L., \& Coelho, J. C. Human bone morphogenetic protein-2 use for maxillary reconstruction in cleft lip and palate patients. $J$. Craniofac. Surg., 23(6):1627-33, 2012.

Carreira, A. C.; Alves, G. G.; Zambuzzi, W. F.; Sogayar, M. C. \& Granjeiro, J. M. Bone morphogenetic proteins: Structure, biological function and therapeutic applications. Arc.h Biochem. Biophys., 561:64-73, 2014 a.

Carreira, A. C.; Lojudice, F. H.; Halcsik, E.; Navarro, R. D.; Sogayar, M. C., \& Granjeiro, J. M. Bone morphogenetic proteins: Facts, challenges, and future perspectives. J. Dent. Res., 93(4):335-45, 2014b.
Chin, M.; Ng, T.; Tom, W. K., \& Carstens, M. Repair of alveolar clefts with recombinant human bone morphogenetic protein (rhbmp-2) in patients with clefts. J. Craniofac. Surg., 16(5):778-89, 2005.

Chung, V. H.; Chen, A. Y.; Jeng, L. B., \& Lee, D. H. Engineered autologous bone marrow mesenchymal stem cells: Alternative to cleft alveolar bone graft surgery. J. Craniofac. Surg., 23(5):1558-63, 2012.

Chung, Y. I.; Ahn, K. M.; Jeon, S. H.; Lee, S. Y.; Lee, J. H., \& Tae, G. Enhanced bone regeneration with bmp-2 loaded functional nanoparticlehydrogel complex. J. Control. Release, 121(1-2):91-9, 2007.

Davies, S. D., \& Ochs, M. W. Bone morphogenetic proteins in craniomaxillofacial surgery. Oral Maxillofac. Surg. Clin. North. Am., 22(1):17-31, 2010.

Dickinson, B. P.; Ashley, R. K.; Wasson, K. L.; O'Hara, C.; Gabbay, J.; Heller, J. B., \& Bradley, J. P. Reduced morbidity and improved healing with bone morphogenic protein-2 in older patients with alveolar cleft defects. Plast. Reconstr. Surg., 121(1): 209-17, 2008.

el-Bokle, D.; Smith, S. J.; Germane, N. \& Sharawy, M. New technique for creating permanent experimental alveolar clefts in a rabbit model. Cleft Palate Craniofac. J., 30(6): 542-7, 1993.

Francis, C. S.; Mobin, S. S.; Lypka, M. A.; Rommer, E.; Yen, S.; Urata, M. M., \& Hammoudeh, J. A. Rhbmp-2 with a demineralized bone matrix scaffold versus autologous iliac crest bone graft for alveolar cleft reconstruction. Plast. Reconstr. Surg., 131(5):1107-15, 2013.

Gentile, P.; Chiono, V.; Carmagnola, I. \& Hatton, P. V. An overview of poly(lactic-co-glycolic) acid (plga)-based biomaterials for bone tissue engineering. Int. J. Mol. Sci., 15(3):3640-59, 2014.

Herford, A. S.; Boyne, P. J.; Rawson, R., \& Williams, R. P. Bone morphogenetic protein-induced repair of the premaxillary cleft. J. Oral Maxillofac. Surg., 65(11):2136-41, 2007. 
Issa, J. P.; do Nascimento, C.; Iyomasa, M. M.; Siessere, S.; Regalo, S. C.; Defino, H. L. \& Sebald, W. Bone healing process in critical-sized defects by rhbmp-2 using poloxamer gel and collagen sponge as carriers. Micron, 39(1):17-24, 2008.

Kamaly, N.; Yameen, B.; Wu, J., \& Farokhzad, O. C. Degradable controlledrelease polymers and polymeric nanoparticles: Mechanisms of controlling drug release. Chem. Rev., 116(4):2602-63, 2016.

Kamaly, N.; Fredman, G.; Fojas, J. J. R.; Subramanian, M.; Choi, W., II; Zepeda, K.; Vilos, C.; Yu, M.; Gadde, S.; Wu, J.; Milton, J.; Carvalho Leitao, R.; Rosa Fernandes, L.; Hasan, M.; Gao, H.; Nguyen, V.; Harris, J.; Tabas, I. \& Farokhzad, O. C. Targeted interleukin-10 nanotherapeutics developed with a microfluidic chip enhance resolution of inflammation in advanced atherosclerosis. ACS Nano, 10(5):5280-92, 2016.

Kawamoto, T.; Motohashi, N.; Kitamura, A.; Baba, Y.; Takahashi, K.; Suzuki, S. \& Kuroda, T. A histological study on experimental tooth movement into bone induced by recombinant human bone morphogenetic protein-2 in beagle dogs. Cleft Palate Craniofac. J., 39(4):439-48, 2002.

Kirby, G.; White, L.; Steck, R.; Berner, A.; Bogoevski, K.; Qutachi, O.; Jones, B.; Saifzadeh, S.; Hutmacher, D.; Shakesheff, K. \& Woodruff, M. Microparticles for sustained growth factor delivery in the regeneration of critically-sized segmental tibial bone defects. Materials, 9(4):259, 2016

Kohane, D. S. Microparticles and nanoparticles for drug delivery. Biotech. Bioeng., 96(2):203-9, 2007.

Kumari, A.; Yadav, S. K. \& Yadav, S. C. Biodegradable polymeric nanoparticles based drug delivery systems. Colloids Surf. B, Biointerfaces, 75(1):1-18, 2010.

Lee, J. H.; Woo, D. K.; Kim, T. H.; Kang, J. G.; Yun, J. W.; Park, J. H.; Park, B. W.; Kang, Y. H.; Rho, G. J.; Jang, S. J.; Park, J. S.; Lee, H. C.; Yoon, Y. M.; Hwang, T. S.; Kim, D. R.; Hwang, S. C.; Lee, D. H.; Kim, H. Y.; Oh, S. H. \& Byun, J. H. In vitro and long-term (2-year follow-up) in vivo osteogenic activities of human periosteum-derived osteoblasts seeded into growth factor-releasing polycaprolactone/pluronic f127 beads scaffolds. J. Biomed. Mat. Res. Part A, 105(2):363-76, 2017.

Li, R. H., \& Wozney, J. M. Delivering on the promise of bone morphogenetic proteins. Trends Biotechnol., 19(7):255-65, 2001.

Mayer, M.; Hollinger, J.; Ron, E., \& Wozney, J. Maxillary alveolar cleft repair in dogs using recombinant human bone morphogenetic protein2 and a polymer carrier. Plast. Reconstr. Surg., 98(2):247-59, 1996.

Mostafa, N. Z.; Doschak, M. R.; Major, P. W. \& Talwar, R. Reliable critical sized defect rodent model for cleft palate research. J. Craniomaxillofac. Surg., 42(8):1840-6, 2014.

Nampo, T.; Watahiki, J.; Enomoto, A.; Taguchi, T.; Ono, M.; Nakano, H.; Yamamoto, G.; Irie, T.; Tachikawa, T. \& Maki, K. A new method for alveolar bone repair using extracted teeth for the graft material. $J$. Periodontol., 81(9):1264-72, 2010.

Nguyen, P. D.; Lin, C. D.; Allori, A. C.; Ricci, J. L.; Saadeh, P. B. \& Warren, S. M. Establishment of a critical-sized alveolar defect in the rat: A model for human gingivoperiosteoplasty. Plast. Reconstr. Surg., 123(3):817$25,2009$.

Pillai, O. \& Panchagnula, R. Polymers in drug delivery. Curr. Opin. Chem. Biol., 5(4):447-51, 2001.

Pulapura, S. \& Kohn, J. Trends in the development of bioresorbable polymers for medical applications. J. Biomat. Appl., 6(3):216-50, 1992.

Puppi, D.; Chiellini, F.; Piras, A. M. \& Chiellini, E. Polymeric materials for bone and cartilage repair. Prog. Polym. Sci., 35(4):403-40, 2010.

Quinlan, E.; Lopez-Noriega, A.; Thompson, E.; Kelly, H. M.; Cryan, S. A. \& O'Brien, F. J. Development of collagen-hydroxyapatite scaffolds incorporating plga and alginate microparticles for the controlled delivery of rhbmp-2 for bone tissue engineering. J. Controll. Release, 198:71-9, 2015.

Rengachary, S. S. Bone morphogenetic proteins: Basic concepts. Neurosurg. Focus, 13(6):1-6, 2002

Riehemann, K.; Schneider, S. W.; Luger, T. A.; Godin, B.; Ferrari, M. \& Fuchs, H. Nanomedicine--challenge and perspectives. Angew. Chem.
Int. Edit., 48(5):872-97, 2009

Ruhe, P. Q.; Boerman, O. C.; Russel, F. G.; Spauwen, P. H.; Mikos, A. G. \& Jansen, J. A. Controlled release of rhbmp-2 loaded poly(dl-lactic-coglycolic acid)/calcium phosphate cement composites in vivo. J. Controll. Release, 106(1-2):162-71, 2005.

Schrier, J. A.; Fink, B. F.; Rodgers, J. B.; Vasconez, H. C. \& DeLuca, P. P. Effect of a freeze-dried cmc/plga microsphere matrix of rhbmp-2 on bone healing. A.A.P.S. Pharm. Sci. Tech., 2(3):E18, 2001.

Shi, S.; Cheng, X.; Wang, J.; Zhang, W.; Peng, L. \& Zhang, Y. Rhbmp-2 microspheres-loaded chitosan/collagen scaffold enhanced osseointegration: An experiment in dog. J. Biomat. Appl., 23(4):33146, 2009.

Urist, M. R. Bone: Formation by autoinduction. Science, 150(3698):8939, 1965.

Vilos, C. \& Velasquez, L. A. Therapeutic strategies based on polymeric microparticles. J. Biomed. Biotech., 2012: 672760, 2012.

Vilos, C.; Constandil, L.; Rodas, P. I.; Cantin, M.; Zepeda, K.; Herrera, N. \& Velasquez, L. A. Evaluation of ceftiofur-phbv microparticles in rats. Drug Des., Devel. Ther., 8: 651-66, 2014.

Vilos, C.; Velasquez, L. A.; Rodas, P. I.; Zepeda, K.; Bong, S. J.; Herrera, N.; Cantin, M.; Simon, F. \& Constandil, L. Preclinical development and in vivo efficacy of ceftiofur-plga microparticles. PloS one, 10(4): e0123335, 2015.

Virlan, M. J. R.; Miricescu, D.; Totan, A.; Greabu, M.; Tanase, C.; Sabliov, C. M.; Caruntu, C. \& Calenic, B. Current uses of poly (lactic-co-glycolic acid) in the dental field: A comprehensive review. J. Chem., 2015: 12, 2015.

Vo, T. N.; Kasper, F. K. \& Mikos, A. G. Strategies for controlled delivery of growth factors and cells for bone regeneration. Adv. Drug Deliv. Rev., 64(12):1292-309, 2012.

Walmsley, G. G.; McArdle, A.; Tevlin, R.; Momeni, A.; Atashroo, D.; Hu, M. S.; Feroze, A. H.; Wong, V. W.; Lorenz, P. H.; Longaker, M. T. \& Wan, D. C. Nanotechnology in bone tissue engineering. Nanomed. $N$. B. M., 11(5):1253-63, 2015.

Woo, E. J. Adverse events reported after the use of recombinant human bone morphogenetic protein 2. J. Oral Maxillofac. Surg., 70(4):765-7, 2012.

Wozney, J. M.; Rosen, V.; Celeste, A. J.; Mitsock, L. M.; Whitters, M. J.; Kriz, R. W.; Hewick, R. M. \& Wang, E. A. Novel regulators of bone formation: Molecular clones and activities. Science, 242(4885):152834, 1988.

Xu, Y.; Sun, J. \& Chen, Z. Establishment of a rat model for alveolar cleft with bone wax. J. Oral Maxillofac. Surg., 73(4):733 e1-10, 2015.

Yameen, B.; Vilos, C.; Choi, W. I.; Whyte, A.; Huang, J.; Pollit, L. \& Farokhzad, O. C. Drug delivery nanocarriers from a fully degradable peg-conjugated polyester with a reduction-responsive backbone. Chem. Eur. J., 21(32):11325-29, 2015.

Zhou, A. J.; Peel, S. A. \& Clokie, C. M. An evaluation of hydroxyapatite and biphasic calcium phosphate in combination with pluronic $\mathrm{f} 127$ and bmp on bone repair. J. Craniofac. Surg., 18(6):1264-75, 2007.

\section{Dirección para correspondencia:}

Francisca Uribe

División de Cirugía Oral y Maxilofacial

Facultad de Odontología

Universidad de La Frontera

Manuel Montt 115

Temuco - CHILE

Recibido :12-01-2017

Aceptado:18-02-2017

E-mail: maria.uribe@ufrontera.cl 